\title{
KANN MAN RECHTSTEXTE KULTURELL EINBETTEN?
}

\author{
Alenka KOCBEK \\ Diplomgermanistin und Diplomanglistin (B.Sc) \\ Fakulteta za managment \\ Univerza na Primorskem (Fakultät für Management, Universität Primorska) \\ Cankarjeva ulica 5, 6000 Koper - Slovenija/Slowenien. \\ alenka.kocbek@guest.arnes.si
}

\begin{abstract}
In many aspects, legal translation seems to be an adequate domain for applying the principles of the functionalist approaches to translation, especially the skopos-theory by J. H. Vermeer. Nevertheless, an indiscriminate application of the principle of cultural embeddedness could prove questionable, if not misleading, as it may cause disturbance in the legal communication occurring through the translation act. In some legal transactions, such as e.g. international contracts, involving contracting parties belonging to different nations, only one legal system is adopted as the communication framework (i.e. the governing or applicable law). In such cases, the functionalist principle of cultural embeddedness needs to be applied selectively, i.e. only with respect to some linguistic features of the text, while in a broader sense, as far as the cultural and/or legal foundations of the text are concerned, the source and the target text will have the same reference frame.
\end{abstract}

\begin{abstract}
Pravna besedila se glede na svojo naravo zdijo posebej primerna za uporabo funkcionalističnih prevodoslovnih pristopov, posebej teorije skoposa. Vendar pa se dosledna uporaba načela kulturne vpetosti jezika lahko izkaže kot neprimerna ali celo zavajajoča, ker lahko povzroči motnje v pravnem sporazumevanju, ki poteka preko prevajalskega dejanja. Pri nekaterih pravnih poslih kot npr. pri pogodbah, v katerih so udeležene stranke iz različnih držav, se kot komunikacijski okvir določi en sam pravni sistem (t.i. merodajno ali veljavno pravo). $\mathrm{V}$ takšnih primerih je mogoče načelo kulturne vpetosti uporabiti samo glede na lingvistične vidikih besedila, v širšem obsegu, t.j. kar zadeva kulturno oz. pravno podlago besedila, pa imata izhodiščno in ciljno besedilo isti referenčni okvir.
\end{abstract}

Zusammenfassung: Unter vielen Aspekten scheint sich die Rechtsübersetzung besonders gut zur Anwendung der Prinzipien der funktionalistischen Translationsansätze, besonders der SkoposTheorie von J.H. Vermeer, zu eignen. Jedenfalls könnte sich eine konsequente Durchsetzung des Prinzips der kulturellen Einbettung als unangebracht, sogar als irreführend erweisen, da sie zu Störungen in der durch die Translationshandlung stattfindenden Rechtskommunikation führen kann. In einigen Rechtsgeschäften, wie z.B. in Verträgen, an denen Vertragsparteien aus verschiedenen Staaten beteiligt sind, wird nur ein Rechtssystem als Kommunikationsrahmen definiert (das sog. maßgebende bzw. anwendbare Recht). In solchen Fällen kann man das Prinzip der kulturellen Einbettung nur mit Bezug auf die linguistischen Aspekte des Textes anwenden, in weiterem Ausmaß, d.h. was die Kultur- bzw. Rechtsgrundlage des Textes betrifft, haben aber der Ausgangs- und der Zieltext den selben Referenzrahmen.

\section{Einleitung}




\section{Comparative Legilinguistics vol. 2/2010}

In der interkulturellen Kommunikation stellen Sprachkenntnisse eine der grundsätzlichen Kompetenzen dar, die die Verständigung zwischen Teilnehmern ermöglichen. Diese Kommunikation verläuft entweder in der Sprache einer der teilnehmenden Parteien oder in einer dritten, neutralen Sprache, die als Lingua franca dient. Sprachkenntnisse als Mittel der interkulturellen Kommunikation umfassen also immer auch einen Anteil expliziter oder impliziter Translation.

Die funktionalistischen Ansätze in der Translatologie und vor allem die Skopos-Theorie von J.H. Vermeer legen besonderen Wert auf die Rolle eines eindeutig definierten Zwecks (Skopos) der Translation und betonen die Wichtigkeit eines präzisen Übersetzungsauftrags, die Rolle des Translators als interkulturellen Experten und das Prinzip der kulturellen Einbettung des Ausgangs- und des Zieltextes. Im Rahmen dieser Ansätze wird die Translation als ein interkultureller Transfer gesehen, bei welchem die interkulturellen Unterschiede unbedingt zu berücksichtigen sind. Während in einer multikulturellen Umgebung die Kommunikationssituationen von vielen unterschiedlichen Aspekten der daran beteiligten Kulturen beeinflusst werden, verlangen in der Rechtskommunikation die Rechtssysteme der Ausgangs- und der Zielkultur besondere Aufmerksamkeit. In der Regel wird das Rechtssystem einer der beteiligten Parteien oder, seltener, ein supranationales oder internationales Rechtssystem als Kommunikationsrahmen gewählt.

\section{Das funktionalistische Prinzip der kulturellen Einbettung}

Die Translation als kommunikative Handlung verfolgt einen bestimmten Zweck, der von Vermeer mit dem altgriechischen Terminus Skopos bezeichnet wird. Dieses Ziel definiert die zu verwendenden Übersetzungsmethoden und Strategien für die Produktion einer funktionell adäquaten Translation. Außerdem findet die Translation in konkreten, definierbaren Situationen statt, die zeitlich und örtlich begrenzt sind und Mitglieder verschiedener Kulturen einbeziehen und in bestimmte Kulturumfelder eingebettet sind. Die Sprache ist also ein wesentliches Kommunikationsmittel, jedoch muss sie im Kontext der entsprechenden Kultur gebraucht werden. In ihrem Werk Translation Studies schildert Bassnett die Verbundenheit und gegenseitige Abhängigkeit der Sprache und Kultur mit der folgenden Metapher:

"No language can exist unless it is steeped in the context of culture; and no culture can exist which does not have at its center, the structure of natural language.

Language, then, is the heart within the body of culture, and it is the interaction between the two that results in the continuation of life-energy. In the same way that the surgeon, operating on the heart, cannot neglect the body that surrounds it, so the translator treats the text in isolation from the culture at his peril. " (Bassnett 1991, 14)

Es besteht kein Zweifel, dass Sprache und Kultur tief miteinander verbunden sind, da gerade die einer Sprache zugrunde liegende Kultur die Codes für die Interpretation der mit Hilfe dieser Sprache vermittelten Mitteilungen durch ihre spezifischen Normen und Konventionen definiert. Im Falle der Rechtssprache deckt sich 


\section{Comparative Legilinguistics vol. 2/2010}

"der Körper der Kultur" mit allen Aspekten der die jeweilige Kultur regulierenden und definierenden Rechtsordnung.

Die Beteiligten an der interkulturellen Kommunikation benötigen also nicht nur gute Sprachkenntnisse, sondern müssen auch andere, für die jeweilige Kommunikationssituation relevante Aspekte der betreffenden Kulturen gründlich kennen. Einige von diesen Aspekten sind der folgenden Definition von Kultur von J. H. Vermeer $\mathrm{zu}$ entnehmen "the entire setting of norms and conventions an individual as a member of his society must know in order to be "like everybody" - or to be able to be different from everybody." (Vermeer 1987, 28).

Ferner weist Reiß darauf hin, dass die Normen einen stärkeren präskriptiven Charakter haben (sie bezeichnen, was die Mitglieder einer Kultur tun müssen bzw. nicht tun dürfen) und sind dementsprechend obligatorisch, während Konventionen Verhaltensregeln sind, die sich allmählich durch allgemeinen Konsens herausgebildet haben und die empfohlenen bzw. erwarteten Verhaltensformen in einer Gesellschaft darstellen (vgl. Reiß und Vermeer 1984, 178). Wenn man das vermeersche Konzept der Kultur als eine Sammlung allgemein akzeptierter Normen und Konventionen, die von den Mitgliedern einer Gesellschaft zu befolgen sind, mit folgender Äußerung von Jenkins vergleicht "law has the basic function in society of guiding human behaviour and regulating human relations" (Jenkins 1980, 103), stellt man fest, dass die Normen, die nach Vermeer das Verhalten von Mitgliedern einer Gesellschaft regeln, eigentlich in der Rechtsordnung einer Gesellschaft enthalten sind.

\section{Die Beziehung zwischen Sprache und Recht}

Da sich in der Rechtskommunikation die beteiligten Rechtssysteme durch ihre Bestimmungen und Vorschriften direkt auf konkrete Rechtsgeschäfte auswirken, haben die interagierenden Parteien zu vereinbaren, welches Rechtssystem als Kommunikationsrahmen bestimmt wird. Innerhalb dieses Rahmens müssen dann Rechtskonzepte und -begriffe zwischen Sprachen, Kulturen und Rechtssystemen übersetzt (d.h. kulturell übertragen) werden.

Ein gutes Beispiel solcher Kommunikation bieten internationale Verträge, die als Rechtsgeschäfte per definitionem von der Rechtsordnung des Staates geprägt sind, dessen Recht für den Vertrag als maßgebend bestimmt wird. Ein wesentliches Vertragselement ist also die Bestimmung über das anzuwendende Recht bzw. die Gerichtsstandklausel, die bestimmt, welches Gericht im Falle von sich zwischen den Parteien ergebenden Streitigkeiten zuständig ist. In internationalen Verträgen wird als maßgebendes Recht am häufigsten das Recht des Staates einer der Vertragsparteien bestimmt (es sei denn, die Parteien entscheiden sich für ein internationales Schiedsgericht/Arbitrage oder für ein supranationales oder internationales Recht). Wenn der Vertrag schon beim Abschluss in zwei Sprachen verfasst wurde, wird entweder eine Fassung als verbindlicher bzw. authentischer Text definiert, oder es gelten beide Fassungen parallel als gleichwertig. Im letzteren Fall ist das als maßgebend bestimmte Recht Kulturbasis beider sprachlichen Versionen. Wenn dem Ausgangs- und Zieltext verwandte Rechtssysteme zugrunde liegen, lässt sich das Prinzip der Translation als kulturellen Transfers relativ gut implementieren. Problematisch sind aber die Fälle, in 


\section{Comparative Legilinguistics vol. 2/2010}

denen eine Lingua franca verwendet wird, der als Kulturbasis eine Rechtsordnung zugrunde liegt, die den Rechtssystemen des Ausgangs- und des Zieltextes fremd ist und sich von ihnen sogar wesentlich unterscheidet.

In diesem Zusammenhang weist Gerard-René de Groot, Professor für vergleichendes und privates Rechts an der Universität Maastricht, darauf hin, dass beim Übersetzen von Rechtstexten die Systemgebundenheit der Rechtssprache unbedingt zu berücksichtigen ist: "The language of the law is very much a system-bound language, i.e. a language related to a specific legal system. Translators of legal terminology are obliged therefore to practice comparative law." (de Groot 1998, 21 ff.). Die Rechtssysteme unterscheiden sich gründlich voneinander und es gibt noch keine standardisierte internationale Rechtsterminologie. Jeder Staat (manchmal sogar Regionen in einem Staat) hat seine eigene unabhängige Rechtsterminologie entwickelt, wobei eine multilinguale internationale Rechtsterminologie (wie die des EU-Rechts) nur allmählich im Rahmen supranationaler Rechtssysteme geschaffen wird, wenn sie der Harmonisierung unterzogen werden.

Sandrini weist darauf hin, dass die Übersetzbarkeit der Rechtstexte in direktem Verhältnis zur Verwandtschaft der an der Translation beteiligten Rechtsordnungen steht (vgl. Sandrini 1999, 17). Rechtssysteme existieren unabhängig von den Rechtssprachen, die sie verwenden, und entstehen durch die Mitwirkung gesellschaftlicher und politischer Umstände. Es besteht keine direkte Übereinstimmung zwischen Rechtssystem und Rechtssprache. Ein Rechtssystem kann mehrere Rechtssprachen benutzen (Kanada, die Schweiz, zweisprachige Regionen in Slowenien, Österreich, Italien, Belgien) und in einem Sprachgebiet können verschiedene Rechtsordnungen Anwendung finden, was der Fall bei dem Vereinigten Königreich und den USA ist.

Wenn man Rechtssysteme nach ihren Quellen, ihrem geschichtlichem, sozialen und politischen Hintergrund, dem Grad der Kodifizierung und den spezifischen Rechtsinstituten analysiert, stellt man die Existenz von Rechtskreisen bzw. Rechtsfamilien von verwandten Rechtsordnungen fest, die auf einer gemeinsamen Rechtskultur aufbauen und einen gemeinsamen Rechtsstil aufweisen. So erweisen sich die Rechtsordnungen, die dem sog. kontinentaleuropäischen Recht (Rechtskreis des Civil law) angehören, das den deutschen, nordischen und romanischen Rechtskreis umfasst, als relativ verwandt. Sie haben gemeinsame Grundlagen in der römischen Rechtstradition und sind durch Kodifizierung gekennzeichnet - die wichtigsten Regeln und Vorschriften sind in schriftlichen Rechtsquellen erfasst. Bei diesen Rechtssystemen ist also eine beträchtliche Verbundenheit der jeweils verwendeten Rechtskonzepte zu erwarten. Andererseits sind Rechtssysteme anderer Länder und Kulturen, die anderen Traditionen entstammen, schwierig zu vergleichen, wie z.B. das fernöstliche, islamische, das HinduRecht und letztendlich der angloamerikanische Rechtskreis, der auf Common law, equity und statute law basiert. Innerhalb des angloamerikanischen Rechtskreises ist Common law das geltende Rechtssystem in England, Wales und mit einigen Unterschieden in den USA, während Schottland und Irland wesentlich anders geartete, stark am kontinentalen Recht angelehnte Rechtssysteme haben, ähnlich der Rechtsordnung des USBundesstaates Louisiana, die ihre Grundlage im französischen Recht hat.

Alle diese Unterschiede wirken sich wesentlich auf die Übersetzbarkeit der Rechtstermini aus, da mit einer vollen Äquivalenz von systembedingten Rechtskonzepten 


\section{Comparative Legilinguistics vol. 2/2010}

kaum zu rechnen ist. Gemäß de Groot sollte man in der ersten Phase des Übersetzens von Rechtskonzepten die Bedeutung des $\mathrm{zu}$ übersetzenden ausgangsprachlichen Rechtserminus analysieren. Nachdem man die beteiligten Rechtssysteme verglichen hat, sucht man nach einem gleichwertigen Terminus im zielsprachlichen Rechtssystem. Findet man kein akzeptables Äquivalent wegen Nichtverwandtschaft der Rechtssysteme, dann wird eine der folgenden subsidiären Lösungen gewählt: Man verwendet den ausgangssprachlichen Terminus in seiner originalen oder transkribierten Version, man setzt eine Paraphrase ein oder man bildet einen Neologismus, d. h. man verwendet in der Zielsprache einen Terminus, der in der zielsprachlichen Terminologie noch nicht existiert und ergänzt ihn nach Bedarf durch eine Fußnote (vgl. de Groot 1998, 25).

Der Äquivalenzgrad der Termini hängt von der Verwandtschaft der Rechtsordnungen und nicht der Verwandtschaft der Sprachen ab. Die Verwandtschaft der Sprachen kann manchmal sogar irreführend wirken und das Entstehen von sog. faux amis verursachen, wie z.B. das deutsche Wort Direktor (das eine operative Funktion in einer Handelsgesellschaft bezeichnet) gegenüber dem englischen Terminus director (Mitglied des Direktorenrates - Board of Directors, eine Funktion, die entweder einem Vorstandsmitglied oder einem Aufsichtsratsmitglied entspricht). Wenn man sich für die zu verwendende translatorische Lösung entscheidet, muss man den Kontext, den Zweck (Skopos) and die Texttypologie in Betracht ziehen. Eine ganze Palette von Skopoi ist möglich: von einer Information über den Ausgangstext für einen Adressaten, der der Zielsprache nicht mächtig ist, bis zu einer Übersetzung, die den Status eines authentischen Textes neben dem Ausgangstext haben wird. Nord klassifiziert Übersetzungen in zwei Grundtypen: die dokumentarische Übersetzung, d.h. ein Dokument in der Zielsprache über gewisse Aspekte einer kommunikativen Interaktion, in welcher ein Ausgangskulturabsender mit einem Ausgangskulturpublikum mittels des Ausgangstextes unter Ausgangskulturbedingungen kommuniziert, und die instrumentelle Übersetzung, deren Ziel es ist, in der Zielsprache ein Instrument für eine neue kommunikative Interaktion zwischen einem Ausgangskulturabsender und einem Zielkulturpublikum unter Verwendung (gewisser Aspekte) des Ausgangstextes als Modell zu produzieren (Nord 1997, 47). Für die Translation in einer Rechtsumgebung muss aber die Kategorisierung weiter ausgearbeitet werden. Cao klassifiziert dementsprechend Rechtsübersetzung in drei Kategorien: Übersetzung für normative Zwecke, Übersetzung für informative Zwecke und Übersetzung für generelle juristische oder gerichtliche Zwecke (2007, 10-12). Die Rechtsübersetzung für normative Zwecke entspricht eigentlich Nords Kategorie der instrumentellen Übersetzung, da sie die Produktion von Übersetzungen von nationalen Gesetzen und internationalen Rechtsinstrumenten in zweisprachigen und mehrsprachigen Rechtsordnungen umfasst, wo der Ausgangs- und der Zieltext gleiche Rechtswirkung haben. Diese Art von Texten wird oft in einer Sprache entworfen und dann in eine oder mehrere andere Sprache(n) übersetzt, jedoch wird die Übersetzung als authentisches Rechtsinstrument betrachtet und ist genauso verbindlich wie der Ausgangstext. Šarčević behauptet, dass man in solchen Fällen nicht von einem Ausgangs- und einem Zieltext sprechen kann, sondern von Paralleltexten im Sinne von authentischen, mehrsprachigen Texten desselben Rechtsinstruments (1999, 104 ff.). Beispiele solcher Übersetzungen sind Rechtstexte die innerhalb zwei- oder mehrsprachigen Rechtsordnungen übersetzt werden (wie die 


\section{Comparative Legilinguistics vol. 2/2010}

Schweiz, zweisprachige Regionen in Italien, Slowenien, Österreich usw.), sowie auch mehrsprachige Rechtsinstrumente der Vereinigten Nationen oder der EU und privatrechtliche Instrumente, wie z. B. Verträge, die in zwei oder mehreren gleichwertigen sprachlichen Versionen entstehen. Gemäß Cao kann Nords Kategorie der dokumentarischen Übersetzung in zwei Subkategorien unterteilt werden. Die erste ist die Rechstübersetzung für informative Zwecke, die deskriptive und konstative Funktionen hat und unterschiedliche Kategorien von Rechtstexten umfasst (Gesetze, Gerichtsentscheidungen, juristische akademische Texte, usw.), die mit dem Ziel verfasst werden, eine Information (in der Form eines Dokumentes) über den Ausgangstext Adressaten in der Zielkultur zu erteilen, wo aber die Übersetzungen einen bloß informativen Wert und keine Rechtskraft haben. Beispiele solcher Übersetzungen findet man in einsprachigen Rechtsordnungen, wenn Texte aus anderen Rechtssystemen übersetzt werden, um als Informationsquelle über fremde Rechtsordnungen zu dienen. Die zweite Subkategorie ist die Übersetzung für generelle oder gerichtliche Zwecke, wo in der Ausgangssprache verfasste Originaltexte übersetzt werden, um vor Gericht, bzw. in Prozessverfahren als Teil der dokumentarischen Beweise benutzt zu werden. Diese Übersetzungen haben sowohl eine informative als auch eine deskriptive Funktion und können neben Rechtsdokumenten (Verteidigungsschriften, Klagebegründungen, Verträgen, usw.) auch gewöhnliche Texte, wie z. B. geschäftliche oder persönliche Korrespondenz, Zeugenaussagen, Berichte von Sachverständigen usw. umfassen, die oft nicht von Juristen und auch nicht in der Rechtssprache verfasst werden, aber wegen spezieller Bedürfnisse der Rechtsübersetzung in die Sphäre der Rechtskommunikation eintreten. Diese Übersetzungen sind für den Gebrauch seitens der Parteien in Gerichtsverfahren gemeint, die der Sprache des Gerichtes nicht mächtig sind.

Erfahrene Übersetzer können in der Regel feststellen, welche Art von Übersetzung in einer gegebenen Rechtsumgebung und in einer bestimmten Kommunikationssituation erforderlich ist, d. h. sie sind in der Lage, den Skopos selbst zu identifizieren. Natürlich kann aber auch der Übersetzungsauftrag diese Informationen enthalten. Gemäß der Skopos-Theorie kann der Übersetzungsauftrag erheblich zur Qualität und Funktionalität der Übersetzung beitragen, und zwar dadurch, dass darin dem Übersetzer explizite oder implizite Auskunft über die intendierte Funktionen des Zieltextes, dessen Adressat(en), die voraussichtliche Zeit, den Ort und das Motiv für die Produktion und die Rezeption des Textes gegeben wird (Nord 1997, 137). Für eine Rechtsübersetzung ist jedoch auch eine Information über die als Kommunikationsrahmen zu berücksichtigende Rechtsordnung notwendig.

\section{Englisch als Lingua Franca der Rechtskommunikation}

Ungeachtet ihrer Herkunft wählen Teilnehmer in der internationalen Rechtskommunikation heute oft Englisch als Sprache ihrer Kommunikation. Der weit verbreitete Gebrauch des Englischen als Lingua franca ist mit Sicherheit dessen Aufstieg als Weltsprache zuzuschreiben (Crystal 1997, 8-10). Diesbezüglich deutet Van Essen (2002, 13) darauf hin, dass Englisch als Lingua franca am häufigsten verwendet wird, nicht um mit Muttersprachlern zu verkehren, sondern als Eintrittsbedingung für eine internationale Gesellschaft von Experten (Wissenschaftler, Juristen, Geschäftsleute), um 


\section{Comparative Legilinguistics vol. 2/2010}

unter Anwendung des Sprachregisters dieser Gemeinschaft über Themen von gemeinsamem Interesse zu kommunizieren. Eine solche Kommunikation findet oft zwischen Nichtmuttersprachlern vom Englischen statt, deren kultureller Hintergrund weder englisch noch amerikanisch ist. Die traditionellen, die britische bzw. amerikanische Kultur betreffenden Kulturkenntnisse erweisen sich in solchen Fällen als völlig nutzlos, andererseits sind aber spezifische Aspekte der beteiligten Kulturen äußerst relevant, deswegen ist spezialisiertes Wissen über solche Aspekte (wie z. B. über Rechtsordnungen in der Rechtskommunikation) eine wichtige Voraussetzung für eine effektive Verständigung. Van Essen $(2002,14)$ deutet in diesem Zusammenhang auf die bisherigen Versuche hin um gemeinsame linguistische Standards (Aussprache, Grammatik, Wortschatz) für die Lingua franca festzusetzen, wie z. B. das Projekt des Englischen als Lingua franca für Europa bzw. ELFE (vgl. dazu Labrie und Quell 1997, Jenkins und Seidlhofer 2001), das in der EU von einigen Sprachexperten gefördert wird mit dem Ziel, den Gebrauch des Englischen in der EU zu standardisieren.

Die obigen Standards für den Gebrauch des Englischen als Lingua franca berücksichtigen hauptsächlich dessen linguistische Dimensionen. Als problematisch erweisen sich aber kommunikative Interaktionen im Englischen, die Kulturelemente der spezifischen soziokulturellen Umgebungen der Interagierenden einbeziehen, welche der angloamerikanischen Kultur fremd sind und trotzdem im Englischen vermittelt werden müssen. Während es sicher nicht leicht ist, linguistische Standards fürs Englische als Lingua franca zu entwickeln, scheint es fast unmöglich, eine gemeinsame Kulturbasis zu entwickeln, auf die man sich in solchen Interaktionen berufen könnte. Das gilt besonders für Kulturaspekte, die so präzise definiert werden müssen wie eine Rechtsordnung und die wegen ihrer äußerst empfindlichen Natur einen extrem genauen und eindeutigen Sprachgebrauch verlangen. Zur Zeit ist Englisch die allgemein akzeptierte Lingua franca der internationalen Rechtskommunikation, die aber eine sehr vorsichtige Anwendung des Prinzips der kulturellen Einbettung verlangt. Wenn man Englisch in Kommunikationssituationen, wo Teilnehmer aus kontinentalen Rechtsystemen interagieren, unter konsequenter Bindung an das angloamerikanische Rechtssystem verwendet, bringt das die potentielle Gefahr mit sich, dass man Rechtskonzepte hereinbringt, die den Kulturen der kommunizierenden Parteien fremd sind, was die Rechtssicherheit der Interaktion beeinträchtigen kann. Leider enthalten sehr wenige Wörterbücher der Rechtssprache genügend Informationen und Hinweise in diesem Sinne, die den Anwender von diesen potentiellen Problemen bzw. Fallen in Kenntnis setzten würden.

\section{Die Dichotomie zwischen Kontinentalem Recht und Common Law}

In der vergleichenden Rechtswissenschaft wurde die Dichotomie zwischen Civil law bzw. kontinentalem Recht und Common law, welches nicht auf geschriebenen, kodifizierten Rechtsquellen basiert, gründlich bearbeitet. Die wichtigsten Quellen des angloamerikanischen Rechtssystems sind Common law, equity und statute law. Common law wird oft als judge-made law d. h. Richterrecht bezeichnet, da es nicht auf schriftlichen Quellen basiert, sondern auf Präzedenzfällen, d. h. Richterentscheidungen, die in früheren Rechtssachen getroffen wurden. Andererseits ist equity ein Terminus, der 


\section{Comparative Legilinguistics vol. 2/2010}

sich auf ein System von Regeln bezieht, das zusätzlich zu Common law angewendet wird und keine direkte Entsprechung in den kontinentalen Rechtsordnungen hat. Eine wichtige Komponente des angloamerikanischen Rechtssystems ist letztendlich statute law, was eigentlich das geschriebene Recht wie z. B. Acts of Parliament bezeichnet, d. $\mathrm{h}$. diejenigen Rechtsquellen, die auch in der angloamerikanischen Rechtsordnung in geschriebener Form vorhanden sind.

Cao $(2007,23)$ behauptet, dass jede Rechtssprache die Geschichte, Evolution und Kultur des entsprechenden Rechtssystems reflektiert. Genauer gesagt, sind im Stil der einzelnen Rechtssprachen die entsprechende Rechtskultur und -logik reflektiert. De Cruz $(1999,91)$ konstatiert u. a., dass der Stil deutscher Rechtstexte die systematische und logische Entwicklung des deutschen Rechts wiedergibt, welches auf höchst abstraktem, systemorientiertem, deduktivem Denken basiert und nicht für Laien, sondern für Experten gemeint ist, die seine Präzision und Gedankenschärfe zu schätzen wissen (vgl. Zweigert and Kötz 1992, 150).

Die Unterschiede und Abweichungen zwischen Common law und kontinentalem Recht resultieren oft aus der Nichtäquivalenz der Termini und Konzepte, die in diesen zwei größten Rechtskreisen verwendet werden und hauptsächlich drei terminologische Felder betreffen (vgl. Cao 2007, 60 ff.), und zwar die Termini, die man zur Bezeichnung verschiedener Rechtsberufe verwendet, die Terminologie, die die verschiedenen Gerichtsstrukturen bezeichnet und die Begriffe, die sich auf spezielle Rechtsgebiete und institutionen beziehen.

Im Bereich der Rechtsberufe hat der vom Staat bevollmächtigte Experte, der Kunden in Rechtssachen berät und sie vor Gericht vertritt, der auf Deutsch Rechtsanwalt genannt wird, im Slowenischen odvetnik heißt und eine fundamentale Rolle in jeder kontinentalen Rechtsordnung hat, keine direkte Entsprechung in angloamerikanischem Recht, so dass man diesen Begriff mit lawyer, counsel, advocate, attorney, solicitor, barrister oder counsellor übersetzen kann. In den USA bezeichnet man einen Rechtsanwalt am häufigsten als lawyer und attorney, oder formeller attorney-at-law, wobei sie alle Rechtssachen vor Gericht führen dürfen. Im Vereinigten Königsreich, Kanada und Australien und in einigen anderen Common law-Rechtssystemen werden die Rechtsanwälte entweder barristers (die zum Auftritt vor einem höheren Gericht bzw. zur Prozessführung bevollmächtigt sind) oder solicitors (die in der Regel die Kunden beraten und nur vor einem niedrigeren Gericht auftreten dürfen) genannt, während in schottischem Recht der Terminus advocate verwendet wird.

Ein weiteres Feld, in welchem terminologische Probleme wegen Unterschiede zwischen Rechtsordnungen entstehen, ist die sich auf Gerichtsbeamten und Richter beziehende Terminologie. In England und Australien werden die Begriffe judge and justice, as well as magistrate (für magistrate courts) verwendet. In Deutschland und Slowenien unterscheidet man zwischen professionellen Richtern, die eine juristische Ausbildung haben (slowenisch sodnik) und Schöffen (slowenisch porotnik), die als ehrenamtliche Richter berufen werden und eigentlich juristische Laien sind, die den professionellen Richtern beistehen und keine Entsprechung in der angloamerikanischen Rechtsordnung haben.

Eine wichtige Quelle von Übersetzungsproblemen sind auch die Termini, die die Struktur und Hierarchie der Gerichte beschreiben. Im englischen Common law werden 


\section{Comparative Legilinguistics vol. 2/2010}

zwei Wörter in diesem Zusammenhang benutzt: der generelle Begriff court und das Wort tribunal für Organe bzw. Foren, die Verwaltungs- bzw. halbgerichtliche Funktionen wahrnehmen, während im Deutschen und Slowenischen nur ein Terminus (Gericht bzw. slowenisch sodišče) verwendet wird. In England umfasst die Gerichtshierarchie die folgenden Ebenen - the House of Lords als höchstes Berufungsgericht, the Supreme Court of Judicature, the Court of Appeal, the High Court of Justice, the Crown Court, the County Courts und the Magistrates Courts. Diese Struktur lässt sich kaum mit der deutschen vergleichen, die vier hierarchische Ebenen umfasst, und zwar das Amtsgericht, das Landesgericht, das Oberlandesgericht und als höchstes Berufungsgericht den Bundesgerichtshof. Das slowenische Gerichtssystem ist dem deutschen ähnlich (die entsprechenden Gerichte sind okrajno, okrožno, višje und vrhovno sodišče), aber so wie die Mehrheit der kontinentalen Gerichtssysteme weist es kaum Ähnlichkeit mit der Gerichtsstruktur des Common law auf.

Der letzte Bereich, wo die systembedingten Unterschiede stark ausgeprägt sind, sind die Terminologiefelder, die spezielle Rechtsbereiche und Institutionen bezeichnen. Innerhalb des kontinentalen Rechtskreises findet man dieselben Hauptdomänen des Rechts in allen Ländern: das Verfassungsrecht, das Verwaltungsrecht, öffentliches internationales Recht, Strafrecht, Prozessrecht, Zivilrecht, Handelsrecht und Arbeitsrecht. Wenn man aber diese Rechtsdomänen mit denjenigen des Common law vergleicht, stellt man viele konzeptuelle und strukturelle Unterschiede fest. Es gibt Rechtsinstitute im kontinentalen Recht, die dem Common law vollkommen fremd sind, wie z. B. causa (Ursache). Andererseits haben viele Konzepte des Common law wie z. B. consideration oder estoppel im Vertragsrecht oder der Begriff privity in verschiedenen Rechtskontexten keine Entsprechung in kontinentalen Rechtssystemen. Ein extrem wichtiges Konzept im kontinentalen Recht, besonders im deutschen und romanischen Rechtskreis, welches jedoch kein Äquivalent im Common law hat, ist das Obligationenrecht, das sich im Laufe von Jahrhunderten auf der Grundlage von Elementen des römischen Rechts entwickelt hat. In ähnlicher Weise hat die englische Rechtsstruktur equity kein entsprechendes Pendant im kontinentalen Recht, und die meisten dazu gehörenden Konzepte und Vorschriften stellen ein Unikum dar.

Gesellschaftsrecht ist eine weitere Domäne, wo die Unterschiede zwischen den zwei Systemen sehr deutlich sind. Das angloamerikanische Gesellschaftsrecht unterscheidet nicht zwischen den Kategorien von Kapitalgesellschaften (slowenisch kapitalske družbe) und Personengesellschaften (slowenisch osebne družbe), sondern nur zwischen incorporated companies, die den Status einer juristischen Person haben und unincorporated companies, die keine Rechtspersönlichkeit besitzen.

Die Termini public limited company und limited liability company kann man mit relativer Sicherheit als Entsprechungen für die Gesellschaftsformen Aktiengesellschaft (slowenisch delniška družba und Gesellschaft mit beschränkter Haftung (slowenisch družba z omejeno odgovornostjo) betrachten, es gibt aber keine vollkommen äquivalente Bezeichnungen in der englischen Rechtsterminologie für Gesellschaftsformen wie Offene Handelsgesellschaft (slowenisch družba $z$ neomejeno odgovornostjo) oder Kommanditgesellschaft (slowenisch komanditna družba).

Andere Beispiele der Nichtäquivalenz beziehen sich auf die unterschiedlichen Verwaltungssysteme in den Aktiengesellschaften - das angelsächsische einstufige (one- 


\section{Comparative Legilinguistics vol. 2/2010}

tier) und das kontinentaleuropäische zweistufige (two-tier) System. Das einstufige System hat nur ein Verwaltungsorgan, den Direktorenrat (the board of directors), während im zweistufigen System zwei Verwaltungsorgane bestehen - der Vorstand (management board, slowenisch uprava) und der Aufsichtsrat (supervisory board, slowenisch nadzorni svet).

Die rechtssystembedingten Übersetzungsprobleme muss man auch innerhalb der EU konfrontieren, wo Englisch die meistverwendete Lingua franca ist (vgl. Kjaer 1999, 72). Wenn Englisch verwendet wird, um Aspekte und Konzepte des EU-Rechts oder der nationalen kontinentaleuropäischen Rechtssysteme zu beschreiben, werden oft Termini benutzt, die durch die ihnen im angloamerikanischen Rechtssystem zugeschriebene Bedeutung gefärbt sind. Wenn man z. B. das kontinentale Konzept bona fides ins Englische übersetzt, wird am häufigsten der Ausdruck good faith benutzt, welcher jedoch dem kontinentalen Begriff nicht ganz gleichwertig ist. Das englische Konzept bezieht die Idee der Fahrlässigkeit nicht ein, während die kontinentale Interpretation von bona fides oft grobe Fahrlässigkeit mit bad faith (Bösgläubigkeit) gleichstellt.

\section{Schlussbemerkungen}

Trotz der Probleme, die der Einbettung der englischen Sprache in der angloamerikanischen Kultur entstammen, wird Englisch sicher die meistverwendete Lingua franca in der internationalen Kommunikation bleiben. Dank der Sprachpolitik der EU, die die Rolle der Sprachen aller Mitgliedstaaten fördert, werden aber andere Sprachen sicher auch vorankommen. Durch die Kontakte des Vereinigten Königsreichs mit anderen Mitgliedstaaten der EU und die Beteiligung an zahlreichen europäischen Institutionen sind auch viele euroenglische Termini, d. h. englische Übersetzungen von kontinentalen Rechtskonzepten auch in den Wortschatz englischer Muttersprachler eingegangen. Langfristig kann man erwarten, dass English als Lingua franca innerhalb der EU zur Entstehung einer gemeinsamen Kulturbasis beitragen wird, d. h. zur Schaffung von Elementen einer gemeinsamen, von allen Benutzern dieser Sprache geteilten europäischen Kultur. Im Bereich der Rechtskommunikation, wo English als Fachsprache benutzt wird, sollten sich die kommunizierenden Parteien bei der Übertragung von Rechtskonzepten der Komplexität dieser translatorischen Aufgaben bewusst sein und das Prinzip der kulturellen Einbettung der Sprache selektiv und unter der Berücksichtigung potentieller Abweichungen zwischen der Sprache und der ihr zugrunde liegenden Rechtsordnung anwenden. In diesem Kontext sollte sicher die Möglichkeit der Verwendung von Sprachen (z. B. des Deutschen) in Betracht gezogen werden, die eine Kulturverwandtschaft im Sinne von Einbettung in vergleichbaren Rechtsordnungen aufweisen. Die Leitlinien bei der Wahrnehmung dieser komplexen translatorischen Aufgaben sollten jedenfalls „die Wahrung der Rechtssicherheit des Zieltextes sowie die Transparenz des translatorischen Handelns“ (Sandrini 1999, 39) sein.

\section{Literatur}

Bassnett, Susan. 1991. Translation studies: Revised edition. London and New York: Routledge. 


\section{Comparative Legilinguistics vol. 2/2010}

Cao, Deborah. 2007. Translating Law. Clevedon: Multilingual Matters Ltd.

Crystal, David. 1997. English as a Global Language. Cambridge: CUP.

De Cruz, Peter. 1999. Comparative Law in a Changing World. London: Cavendish Publishing.

Essen van, Arthur J. 2002. English is not English. Babylonia 1/02: 10-15.

Groot de, Gerard-René. 1998. Language and Law. In Netherlands reports to the fifteenth international congress of comparative law. 21 -32. Antwerp/Groningen: Intersentia.

Jenkins, Iredell. 1980. Social Order and the Limits of Law: A theoretical Essay. Princeton NY: Princeton University Press.

Jenkins, Jennifer, und Barbara Seidlhofer. 2001. Be proud of your lingua franca. The Guardian Weekly, April 19.

Kjær, Anne Lise. L. 1999. Überlegungen zum Verhältnis von Sprache und Recht bei der Übersetzung von Rechtstexten der Europäischen Union. In Übersetzen von Rechtstexten: Fachkommunikation im Spannungsfeld zwischen Rechtsordnung und Sprache, Hrsg. Peter Sandrini, 63-79. Tübingen: Narr.

Labrie, Normand, und Carsten Quell. 1997. Your language, my language or English? The potential language choice in communication among nationals of the European Union. World Englishes 16 (1): 3-26.

Nord, Christiane. 1997. Translating as a purposeful activity: Functionalist approaches explained. Manchester: St. Jerome.

Reiß, Katharina, und Hans J. Vermeer. 1984. Grundlegung einer allgemeinen Translationstheorie. Tübingen: Niemeyer.

Sandrini, Peter. 1999. Translation zwischen Kultur und Kommunikation: Der Sonderfall Recht. In Übersetzen von Rechtstexten. Fachkommunikation im Spannungsfeld zwischen Rechtsordnung und Sprache, Hrsg. Peter Sandrini, 9-43. Tübingen: Narr.

Šarčević, Susan. 1999. Das Übersetzen normativer Rechtstexte. In Übersetzen von Rechtstexten. Fachkommunikation im Spannungsfeld zwischen Rechtsordnung und Sprache, Hrsg. Peter Sandrini, 103-118. Tübingen: Narr.

Vermeer, Hans J. 1987. What does it mean to translate? Indian Journal of Applied Lingustics 13 (2): 25-33.

Zweigert, Konrad, und Hein Kötz. 1992. An Introduction to Comparative Law. Oxford: Clarendon Press. 
